\title{
Tissue Factor Pathway Inhibitor-1 Is a Valuable Marker for the Prediction of Deep Venous Thrombosis and Tumor Metastasis in Patients with Lung Cancer
}

\author{
Xianming Fei, Huan Wang, Wufeng Yuan, Mingyi Wo, and Lei Jiang \\ Center of Laboratory Medicine, Zhejiang Provincial People's Hospital, Hangzhou 310014, China \\ Correspondence should be addressed to Xianming Fei; fortunity@163.com
}

Received 17 September 2016; Revised 23 December 2016; Accepted 9 January 2017; Published 26 January 2017

Academic Editor: Noriyoshi Sawabata

Copyright @ 2017 Xianming Fei et al. This is an open access article distributed under the Creative Commons Attribution License, which permits unrestricted use, distribution, and reproduction in any medium, provided the original work is properly cited.

Activation of blood coagulation contributes to cancer progression. Tissue factor pathway inhibitor-1 (TFPI-1) is the main inhibitor of extrinsic coagulation pathway. The aim of this study is to assess the predicting significance of TFPI-1 for thrombotic complication and metastasis in lung cancer patients. Total of 188 non-small cell lung cancer (NSCLC) patients were included in this study. Plasma TFPI-1, D-dimer (D-D), antithrombin (AT), Fibrinogen (Fbg), and coagulating factor VIII activity (FVIII:C) were measured. In NSCLC patients, significantly decreased TFPI-1 and AT and increased D-D, Fbg, and FVIII:C levels were observed, and there was a significant correlation between TFPI- 1 and other hemostatic parameters $(P<0.001$, resp.). NSCLC patients with deep venous thrombosis (DVT) or metastasis had significantly lower TFPI-1 levels than those without DVT or metastasis $(P<0.01$, resp.). Multivariate regression revealed that TFPI-1 acted as a predictor for DVT or tumor metastasis in NSCLC patients [OR: 4.15 or 3.28, $P<0.05$, resp.]. The area under ROC curve of TFPI-1 was 0.905 (95\% CI, 0.842 0.967) or 0.828 (95\% CI, 0.742 0.915) for predicting DVT or metastasis $(P<0.001$, resp.). The optimal point of TFPI-1 was 57.7 or $54.3 \mathrm{ng} / \mathrm{mL}$ for predicting DVT or metastasis, respectively. Combination of TFPI-1 and D-D measurements can improve the predicting power for DVT or metastasis in NSCLC patients. Our findings suggested that TFPI-1 was a valuable predictor of DVT and tumor metastasis in NSCLC patients.

\section{Introduction}

Many individuals with cancer are also in a hypercoagulable state [1]. Disorder of blood coagulation and hypercoagulativity may greatly increase the overall risk of thrombosis and even lead to thrombotic events in malignancy patients $[2,3]$. Thrombosis is a frequent complication of malignant diseases which is also associated with short survival [4]. Therefore, serious thrombotic complications such as deep venous thrombosis (DVT) are the second leading causes of death in patients with cancer [5]. As we know, lung cancer patients may exhibit more serious disorder of blood coagulation and higher risk of DVT than other malignancy patients. And DVT would more easily result in tumor metastasis and recurrence of lung cancer [6]. Therefore, lung cancer patients have higher incidence of morbidity and mortality [7]. Coagulation inhibitors play important roles in preventing individuals from thrombosis and, at least in part, dysfunctional blood coagulation in cancer patients, which might be the results of inadequate activity of coagulation inhibitors [8].

TFPI, a potent anticoagulant protein, including TFPI-1 and TFPI-2, would inhibit the activity of the TF-factor/VIIa catalytic complex which is involved in activation of IX to IXa and also could directly inhibit FXa which activated blood coagulation. Both endothelial cells and platelets would produce TFPI-1 [9]. Low plasma TFPI-1 levels have been reported in patients with ischemic stroke and thrombotic thrombocytopenic purpura and in women taking combined oral contraceptives [10]. Much studies demonstrated that lower plasma TFPI-1 levels were associated in individuals with venous and arterial thrombotic disease [11]. And tumor cells expressed large quantities of procoagulant molecules in particular tissue factor (TF), which may enhance malignant growth or invasion [12]. But for the inhibitor of TFfactor/VIIa, there were little studies focus on the relationship 
between TFPI-1 and the progression of lung cancer. Because non-small cell lung cancer (NSCLC) constitutes $85 \%$ of lung cancer [13], the aim of this study was to explore the clinical prediction of TFPI-1 for NSCLC patients with DVT and tumor metastasis.

\section{Materials and Methods}

2.1. Patients Population. A total of 188 NSCLC patients, including 118 male and 70 female patients aged 41 to 77 years from the Department of Oncology, Zhejiang Provincial People's Hospital, China, between May 2013 and September 2014, were enrolled in our prospective cohort study. All patients were Hans and diagnosed according to the histological diagnosis criteria. The pretreatment evaluation included detailed clinical history and physical examination, the clinical data of patients during hospitalization, and the incidence of DVT formation and tumor metastasis at the time of diagnosis. Patients with DVT or tumor metastasis were screened through either typical or doubtful symptoms before treatment and were confirmed by subsequent compression ultrasonography or histopathology, respectively. The exclusion criteria were primary liver and kidney dysfunctions, after operation, hypertension, cardiovascular and cerebrovascular diseases, complicating other malignancies, inflammation and infections, and other diseases potentially activating blood coagulation system. A disease control group was composed of 112 age and sex-matched patients excluding extra pulmonary diseases especially the diseases mentioned above and only with benign lung diseases including pneumonia, pulmonary tuberculosis, chronic bronchitis, lung sarcoidosis, emphysema, and silicosis. Another 130 healthy individuals (38-80 years) were used as healthy control group. None of the patients and controls were taking any drugs affecting hemostatic and fibrinolytic parameters (e.g., anticoagulating, antiplatelet, and fibrinolytic drugs, and oral contraceptives) in two weeks before samples collecting. Informed consent was obtained from the patients or their relatives, and the study was approved by the institutional review board of Zhejiang Provincial People's Hospital.

2.2. Laboratory Analysis. Venous blood of all patients was collected in the morning after an overnight fast to avoid the differences of diurnal variation, especially for hemostatic parameters before treatment, respectively. For coagulation and fibrinolysis, blood sample ( 9 vol) was collected into vacutainer tubes (Becton Dickinson, Mountain View, CA, USA) containing $0.129 \mathrm{~mol} / \mathrm{l}$ trisodium citrate $(1 \mathrm{vol})$. Platelet-poor plasma was obtained by centrifugation at $1500 \mathrm{~g}$ at room temperature for 15 minutes. Aliquots of plasma were transferred into plastic tubes without delay and frozen at $-80^{\circ} \mathrm{C}$ until assays for determination of TFPI-1. At the same time, the measurements of plasma D-dimer (D-D), antithrombin (AT), fibrinogen (Fbg), coagulating factor VIII procoagulant activity (FVIII:C), prothrombin time (PT), and activated partial thromboplastin time (aPTT) were performed with a coagulation analyzer (CS-5100, Japan Sysmex) and the commercial reagents (Siemens Healthcare Diagnostics Products
$\mathrm{GmbH}$ ) within 2 hours after samples collection, respectively. The concentration of plasma TFPI-1 was determined using commercially available enzyme-linked immunosorbent assay kit (American Diagnostica). Routine blood and biochemical parameters (including white blood cell and platelet counts, high-sensitive C-reactive protein, and fast serum glucose) were measured. And the biological and clinical data of NSCLC patients (age and sex/Agenda, body mass index, blood pressure, tumor-related, and pathological data) in the duration of hospitalization was collected and reviewed; the incidence of DVT and tumor metastasis at the time of diagnosis was calculated to evaluate the disease conditions of inhospital NSCLC patients. The fast blood samples of healthy control were collected, and TFPI-1 and other parameters were assayed using the same methods.

2.3. Statistical Analysis. Data was analyzed by one way analysis of variance (ANOVA). And statistical analyses were performed by Student's $t$-test for samples of normal distribution data and Mann-Whitney U-test for not normal distribution data. And for categorical variables, Chi-square test was used. Correlations between the levels of TFPI-1 and other parameters were performed with Pearson correlation analysis. Multivariate Logistic regression analysis was performed to calculate the adjusted odds ratio (OR) and the $95 \%$ confidence interval. The receiver operating characteristic (ROC) curve was were constructed, and areas under curves (AUC) were calculated to illustrate the diagnostic power of TFPI-1. $P$ value of less than 0.05 was considered statistically significant.

\section{Results}

The clinical characteristics of NSCLC patients were shown in Table 1 . Overall, about $54 \%$ of the tumors were adenocarcinoma, and $29 \%$ were of the type of squamous-cell. At the time of diagnosis, $62.77 \%$ of patients had stage IIIb or worse state of NSCLC, and the incidence of DVT and tumor metastasis was $21.80 \%$ and $35.11 \%$, respectively. We observed the DVT rate in NSCLC patients with metastasis was significantly higher than those patients without metastasis $(P<0.001)$. The biological and clinical parameters of controls and patients with NSCLC were presented in Table 2. There was no significant difference for levels of the biological parameters in age, gender, smoking status, blood pressure, BMI, Glu, and WBC between the three groups. As expected, CRP level was significantly higher in NSCLC and benign lung diseases groups than that in healthy control group $(P<0.05)$. Compared with the two control groups, Fbg, D-D, and FVIII:C levels were significantly increased in NSCLC patients, whereas AT and TFPI-1 levels significantly decreased $(P<0.01)$. The levels of other parameters including PT, aPTT, WBC, and PLT were not different from the two control groups.

The correlation between plasma TFPI-1 levels and AT, D$\mathrm{D}, \mathrm{Fbg}$, and FVIII:C levels in NSCLC patients was presented in Figure 1. The plasma TFPI-1 levels were positively correlated with AT levels $[r=0.759, P<0.001$; Figure 1(a)]. 
TABLE 1: The clinical characteristics of patients with non-small cell lung cancer.

\begin{tabular}{lcc}
\hline & Number $(n)$ & Proportion $(\%)$ \\
\hline Number & 188 & 54.25 \\
NSCLC type & 102 & 29.25 \\
$\quad$ Adenocarcinoma & 55 & 16.50 \\
$\quad$ Squamous cell & 31 & 42.55 \\
$\quad$ Others & & 57.45 \\
Stage of NSCLC & 80 & 21.80 \\
$\quad$ IIIIb & 108 & 75.61 \\
$\quad$ IIIb & & $24.39^{*}$ \\
Complicating DVT & 41 & 78.20 \\
$\quad$ Yes & 31 & 35.11 \\
$\quad$ With metastasis & 10 & 46.97 \\
$\quad$ Without metastasis & 147 & 53.03 \\
No & & 64.89 \\
Tumor metastasis & 66 & 31 \\
$\quad$ Yes & 35 & 122 \\
$\quad$ With DVT & & \\
$\quad$ Without DVT & &
\end{tabular}

NSCLC: non-small cell lung cancer. DVT: deep venous thrombosis. $P$ value: compared with patients with metastasis, respectively. ${ }^{*} P<0.001$.

TABLE 2: The biological and clinical parameters of controls and patients with NSCLC.

\begin{tabular}{|c|c|c|c|c|}
\hline Variables & NSCLC patients & Disease controls & Healthy controls & $P$ value \\
\hline Number of subjects & 188 & 112 & 130 & \\
\hline Age (years) & $45.2 \pm 12.3$ & $47.1 \pm 11.4$ & $47.9 \pm 13.2$ & $>0.05$ \\
\hline $\operatorname{Sex}(M / F)$ & $118 / 70$ & $61 / 51$ & $72 / 58$ & $>0.05$ \\
\hline BMI $\left(\mathrm{kg} / \mathrm{m}^{2}\right)$ & $25.3 \pm 2.7$ & $26.1 \pm 3.0$ & $25.5 \pm 2.7$ & $>0.05$ \\
\hline Smoking subjects $(\mathrm{M} / \mathrm{F})$ & $94 / 17$ & $45 / 15$ & $60 / 17$ & $>0.05$ \\
\hline $\mathrm{SBP}(\mathrm{mmHg})$ & $130 \pm 15$ & $132 \pm 14$ & $133 \pm 12$ & $>0.05$ \\
\hline $\mathrm{DBP}(\mathrm{mmHg})$ & $82 \pm 10$ & $80 \pm 11$ & $79 \pm 12$ & $>0.05$ \\
\hline Glu (mmol/L) & $5.12 \pm 1.55$ & $5.30 \pm 1.69$ & $5.25 \pm 1.77$ & $>0.05$ \\
\hline $\operatorname{WBC}\left(10^{9} / \mathrm{L}\right)$ & $6.17 \pm 2.22$ & $5.74 \pm 3.01$ & $5.42 \pm 2.15$ & $>0.05$ \\
\hline $\operatorname{PLT}\left(10^{9} / \mathrm{L}\right)$ & $190.1 \pm 21.5$ & $199.3 \pm 18.0$ & $210.1 \pm 20.1$ & $>0.05$ \\
\hline CRP (mg/L) & $5.5(0.5-19.5)^{\triangleleft}$ & $4.5(0.5-14.6)^{\triangle}$ & $2.4(0.5-7.6)$ & $<0.05$ \\
\hline PT (seconds) & $11.0 \pm 3.1$ & $11.6 \pm 2.6$ & $11.7 \pm 2.2$ & $>0.05$ \\
\hline aPTT (seconds) & $24.1 \pm 5.0$ & $25.8 \pm 3.9$ & $26.0 \pm 3.8$ & $>0.05$ \\
\hline Fbg $(g / L)$ & $3.35 \pm 1.20^{*}$ & $2.68 \pm 1.30$ & $2.54 \pm 2.01$ & $<0.01$ \\
\hline $\mathrm{D}-\mathrm{D}(\mathrm{mg} / \mathrm{L})$ & $0.65 \pm 0.14^{*}$ & $0.42 \pm 0.10$ & $0.26 \pm 0.04$ & $<0.01$ \\
\hline FVIII:C (\%) & $107.5 \pm 12.3^{*}$ & $95.1 \pm 11.2$ & $91.2 \pm 13.0$ & $<0.05$ \\
\hline AT (\%) & $77.0 \pm 10.1^{*}$ & $92.4 \pm 10.8$ & $98.5 \pm 13.1$ & $<0.01$ \\
\hline TFPI-1 (ng/ml) & $51.0 \pm 8.6^{*}$ & $79.2 \pm 13.2$ & $87.1 \pm 15.9$ & $<0.01$ \\
\hline
\end{tabular}

Data are presented as mean \pm SD, and CRP is expressed with median and range. NSCLC: non-small cell lung cancer. BMI: body mass index. SBP: systolic blood pressure. DBP: diastolic blood pressure. GLU: serum glucose. WBC: white blood cell count. PLT: platelet count. CRP: C- reactive protein. PT: prothrombin time. aPTT: activated partial thromboplastin time. Fbg: fibrinogen. D-D: d-dimer. FVIII:C: coagulating factor VIII procoagulant activity. AT: antithrombin. TFPI-1: tissue factor pathway inhibitor-1. $P$ value: compared among three groups. ${ }^{*} P<0.01$ : compared with disease controls and healthy controls. $P<0.05$ : compared with healthy controls.

Whereas there was a negative correlation between plasma TFPI-1 levels and D-D, Fbg, and FVIII:C levels $[r:-0.812$, $P<0.001 ; r:-0.814, P<0.001 ; r:-0.771, P<0.001$, resp.; Figures 1(b), 1(c), and 1(d)].
Comparison of TFPI-1 levels in NSCLC patients with DVT or tumor metastasis with ones without DVT or metastasis was presented in Figure 2. In NSCLC patients with DVT or metastasis, plasma TFPI-1 levels were significantly higher 


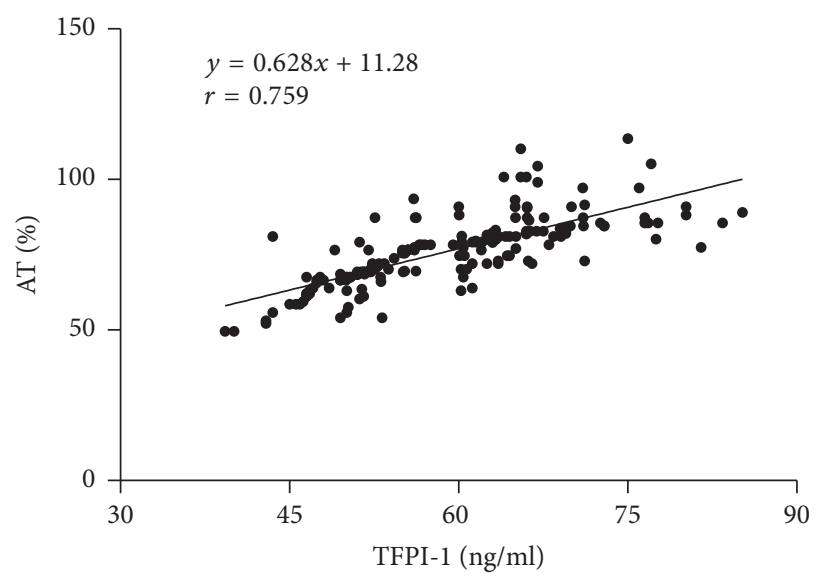

(a)

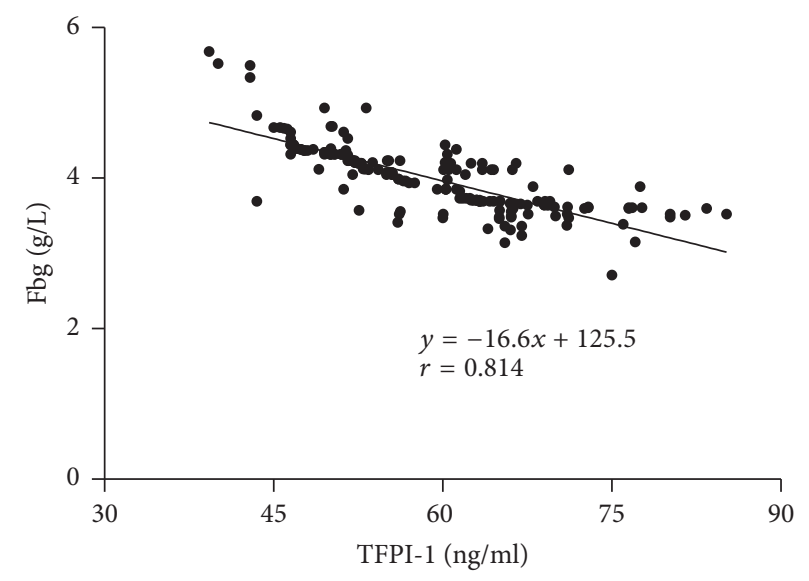

(c)

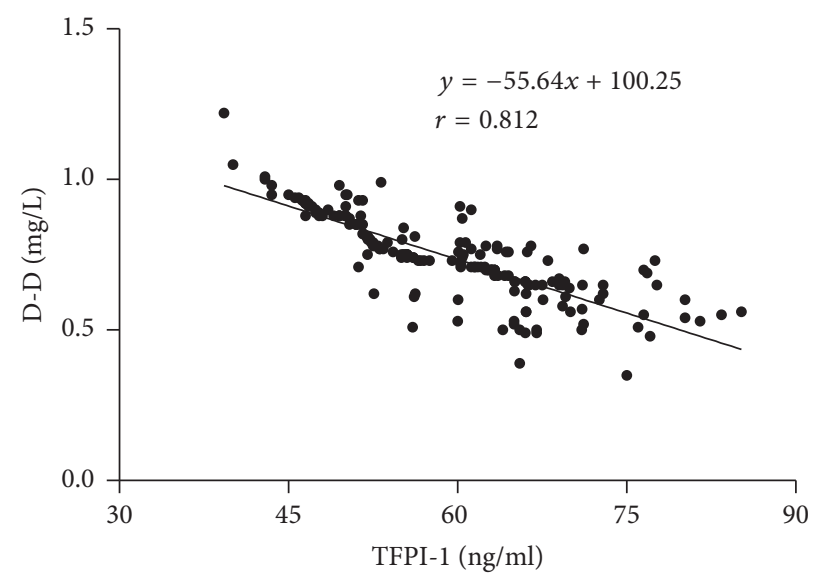

(b)

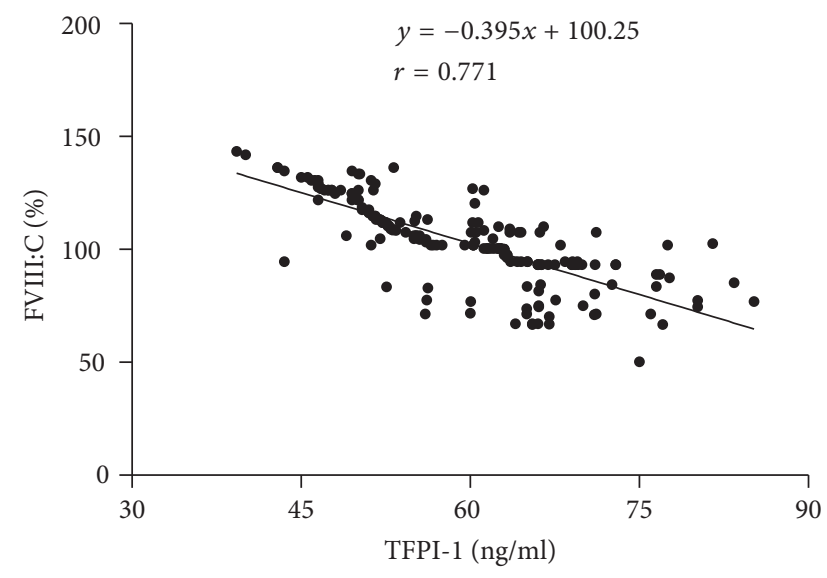

(d)

FIGURE 1: The correlation between plasma TFPI-1 levels and AT, D-D, Fbg, and FVIII:C levels in NSCLC patients. TFPI-1: tissue factor pathway inhibitor-1. AT: antithrombin. D-D: D-dimer. Fbg: fibrinogen. FVIII:C: coagulating factor VIII procoagulant activity.

TABLE 3: Multivariate Logistic regression analysis of risk factors for complicating DVT in NSCLC patients.

\begin{tabular}{lcccc}
\hline Variables & Partial regression coefficient & Standard error & Wald $X^{2}$ & OR (95\% CI) \\
\hline TFPI-1 & 1.35 & 0.55 & 6.22 & $4.15(2.00-8.35)$ \\
AT & 1.14 & 0.38 & 7.29 & $3.22(2.25-5.85)$ \\
Fbg & 0.90 & 0.43 & 8.01 & $2.58(1.15-6.21)$ \\
FVIII:C & 0.75 & 0.50 & 5.55 & $1.80(1.52-6.58)$ \\
D-D & 0.56 & 0.35 & 10.32 & 0.011 \\
\hline
\end{tabular}

NSCLC: non-small cell lung cancer. DVT: deep venous thrombosis. TFPI-1: tissue factor pathway inhibitor-1. AT: antithrombin. Fbg: fibrinogen. FVIII:C: coagulating factor VIII procoagulant activity. D-D: D-dimer. OR: odds ratio. CI: confidence interval.

than that in patients without DVT or metastasis $(t: 24.644$, $P<0.001 ; t: 20.021, P<0.001$, resp.).

Results of multivariate regression analysis of risk factors for complicating DVT or tumor metastasis in NSCLC patients were presented in Tables 3 and 4. Logistic regression analyses showed that TFPI-1 was the independent risk factor related to DVT and tumor metastasis in NSCLC patients (odds ratio, 3.651; 95\% confidence interval, 3.011 4.378, $P=$ 0.00 ; odds ratio, 3.122; 95\% confidence interval, 2.620 3.781, $P=0.00$, resp.).
ROC curves of TFPI-1 for the prediction of DVT or tumor metastasis in NSCLC patients were presented in Figure 3. The ROC curves showed the area under curve of 0.905 (95\% confidence interval, $0.842 \sim 0.967, P<0.001)$ for predicting DVT and 0.828 (95\% confidence interval, 0.742 0.915, $P<$ 0.001 ) for predicting metastasis. The optimal point (cutoff) of TFPI-1 was $57.7 \mathrm{ng} / \mathrm{mL}$ for predicting DVT (sensitivity: $85 \%$, specificity: $68 \%$ ) and $54.3 \mathrm{ng} / \mathrm{mL}$ for predicting metastasis (sensitivity: $80 \%$, specificity: 78\%). ROC curves of TFPI1 combining D-D for the prediction of DVT or tumor 
TABLE 4: Multivariate Logistic regression analysis of risk factors for tumor metastasis in NSCLC patients.

\begin{tabular}{|c|c|c|c|c|c|}
\hline Variables & Partial regression coefficient & Standard error & Wald $X^{2}$ & OR $(95 \% \mathrm{CI})$ & $P$ \\
\hline TFPI-1 & 1.72 & 0.48 & 9.05 & $3.28(2.12-10.98)$ & 0.002 \\
\hline AT & 1.40 & 0.40 & 7.55 & $3.00(2.33-8.65)$ & 0.012 \\
\hline Fbg & 1.15 & 0.60 & 8.50 & $2.61(1.31-8.31)$ & 0.006 \\
\hline FVIII:C & 0.90 & 0.55 & 6.01 & $2.02(1.14-5.11)$ & 0.023 \\
\hline D-D & 0.77 & 0.33 & 11.39 & $1.25(1.07-6.32)$ & $<0.001$ \\
\hline
\end{tabular}

NSCLC: non-small cell lung cancer. TFPI-1: tissue factor pathway inhibitor-1. AT: antithrombin. Fbg: fibrinogen. FVIII:C: coagulating factor VIII procoagulant activity. D-D: D-dimer. OR: odds ratio. CI: confidence interval.

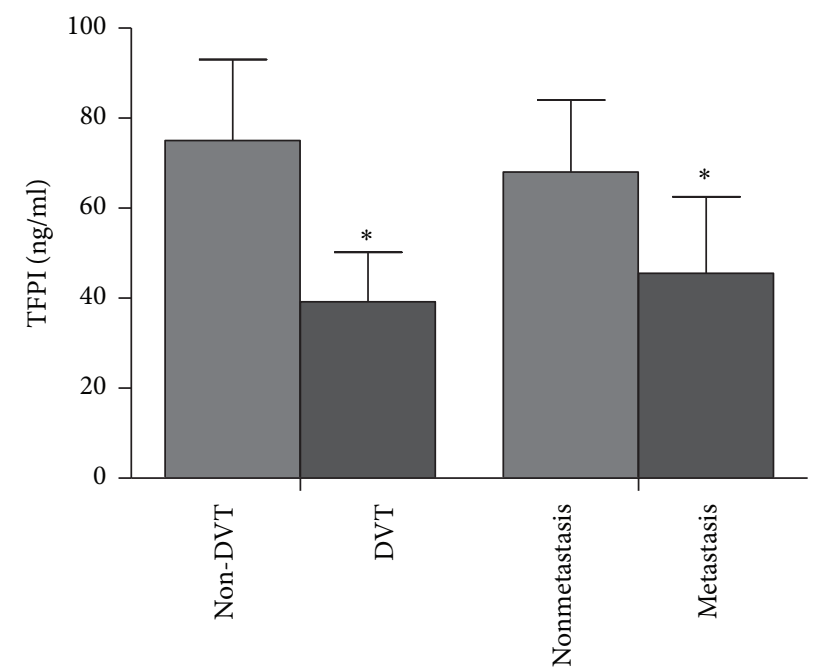

Figure 2: Comparison of TFPI-1 levels in NSCLC patients with different clinical conditions. TFPI-1: tissue factor pathway inhibitor1. DVT: deep venous thrombosis. ${ }^{*} P<0.01$ : compared with nonDVT and nonmetastasis, respectively.

metastasis in NSCLC patients were presented in Figure 4. The ROC curves showed the area under curve of 0.928 (95\% confidence interval, $0.885 \sim 0.985, P<0.001)$ for predicting DVT and 0.878 (95\% confidence interval, 0.805 0.951, $P<$ 0.001 ) for predicting metastasis. The sensitivity was $87 \%$ and $85 \%$, and specificity was $79 \%$ and $70 \%$, respectively.

\section{Discussion}

Tumor cells may activate coagulation system and lead to thrombosis which is mainly through the production of procoagulant, fibrinolytic, and proaggregating activities and the release of proinflammatory and proangiogenic cytokines [14]. Falanga et al. reported that different components of hemostatic system play an important role in tumor metastasis [15]. Therefore, All of the abovementioned causes may be involved in the occurrence of prothrombotic state even DVT and tumor metastasis in patients with cancer. Some reports have showed that there were higher incidences of DVT, tumor dissemination, and metastasis and higher mortality in patients with lung cancer $[14,16]$. In the present study, we found that the incidence of DVT and tumor metastasis before treatment were $21.8 \%$ and $35.1 \%$ in patients with NSCLC, respectively, which were higher than those $11 \%$ or $1 \sim 8 \%$ reported in other studies $[13,17]$. The inconsistent results may be due to difference of population race, the verification of venous thrombosis, and the longer courses of disease before diagnosis of the patients in this study. Another finding was DVT rate of $76 \%$ in NSCLC patients with metastasis, and a metastasis rate of about $47 \%$ in all patients complicating DVT, indicating that DVT is closely associated with tumor metastasis in NSCLC patients.

Antithrombotic system includes many important components, of which AT is the major anticoagulant in plasma of human body [18]. However, TFPI-1 plays more important roles than AT and other anticoagulants involved in the inhibition of extrinsic pathway of blood coagulation, which is the initiating pathway of blood coagulation [19]. Lower TFPI-1 levels were associated with a lot of thrombotic disease [11]. In the present study, NSCLC patients showed higher D-D and Fbg levels and lower AT levels than that in control individuals. Furthermore, there were lower TFPI-1 levels in NSCLC patients, which indicated that hypercoagulable state existed in NSCLC patients, and decreased TFPI-1 levels were in accordance with abnormal levels of the abovementioned thrombotic and hemostatic parameters. Although the mechanisms by which coagulation is activated in cancer is multifactorial, tissue factor (TF) is traditionally recognized to play an important role in this process [20]. Obviously, as the inhibitor of TF, TFPI-1 is an important inhibitor of coagulation activation. Though we have observed decreased TFPI-1 levels in NSCLC patients, our results seem to disprove the reported results of elevated TFPI in solid tumor diseases [21]. Within our knowledge, the following causes may partly explain the difference. In the present study, only NSCLC were included, and other solid tumors were not. Therefore, different TFPI-1 concentration range might result from different tumor type. TFPI includes both TFPI-1 and TFPI-2 and we did not measure TFPI-2 levels. However, TFPI-2 is a highly effective inhibitor of TFmediated coagulation and cellular migration [19], which may be an important reason for the difference. We observed the mean of TFPI-1 levels, but it was the median in the report, which may be the third cause of the difference. And our study also demonstrated that TFPI-1 levels were positively and negatively correlated with AT, D-D, Fbg, and FVIII:C levels, respectively, which further indicated that decreased TFPI-1 was closely associated with blood coagulation abnormalities in NSCLC patients, and lower TFPI-1 may have a lower inhibitory power on initial activation of extrinsic coagulation 


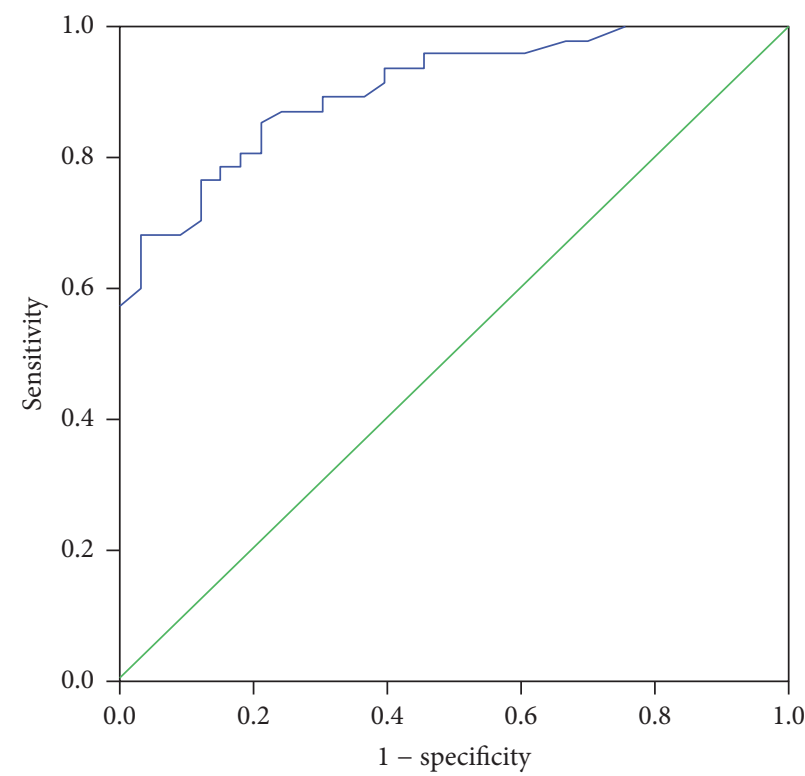

(a)

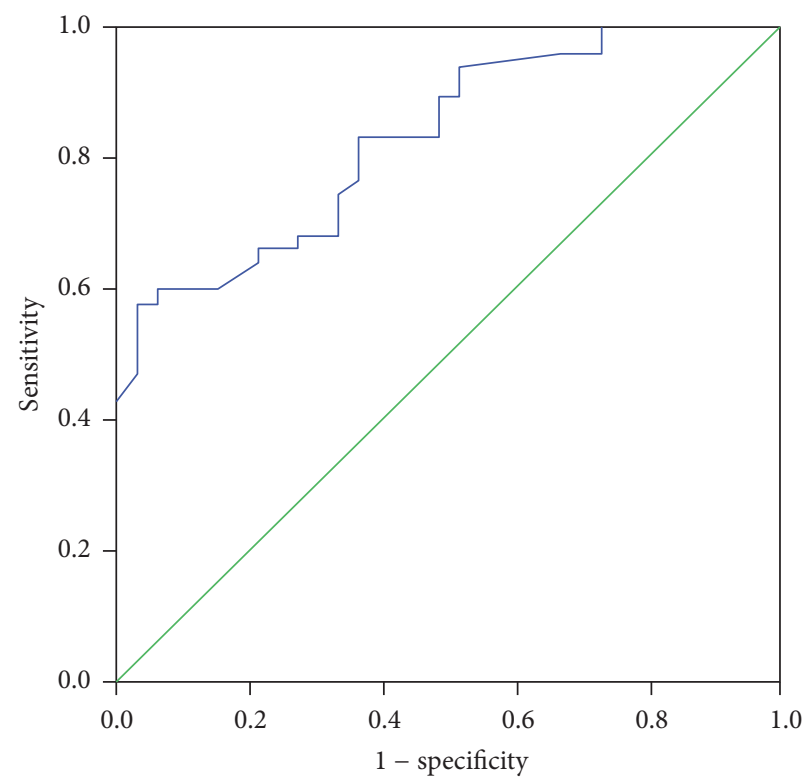

(b)

FIGURE 3: ROC curves of TFPI-1 for predicting DVT and tumor metastasis in NSCLC patients. ROC: receiver operating characteristic. DVT: deep venous thrombosis. (a) ROC curve for DVT. (b) ROC curve for tumor metastasis.

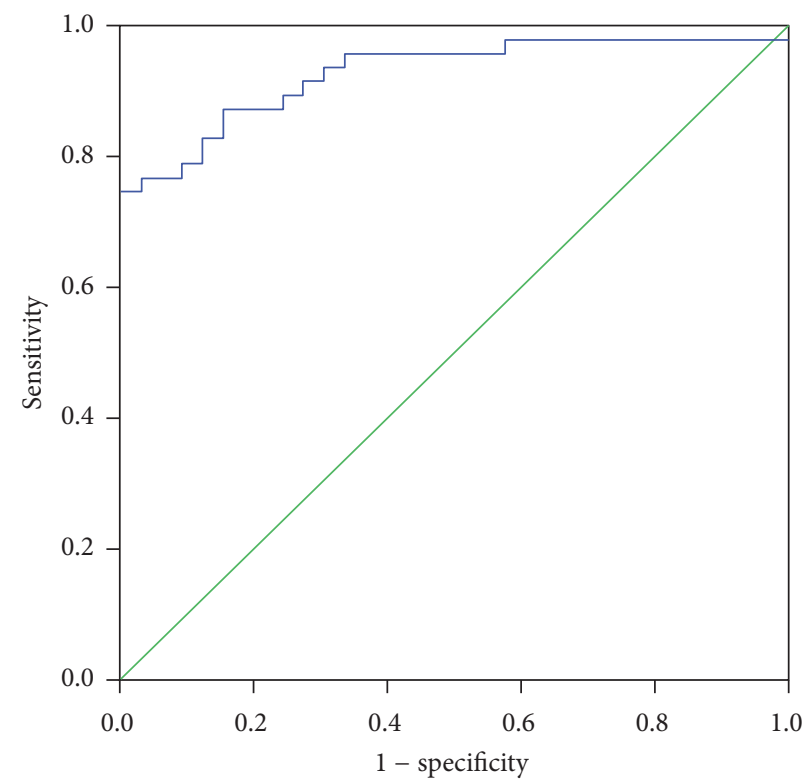

(a)

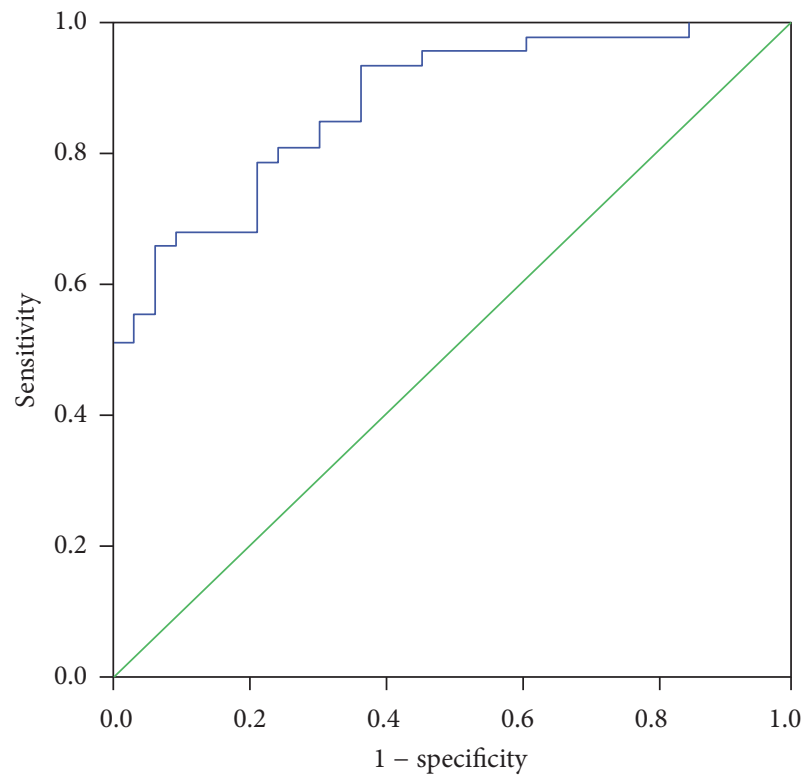

(b)

FIGURE 4: ROC curves of TFPI-1 combining D-dimer for predicting DVT and tumor metastasis in NSCLC patients. ROC: receiver operating characteristic. DVT: deep venous thrombosis. (a) ROC curve for DVT. (b) ROC curve for tumor metastasis.

pathway. Therefore, as a result of lower TFPI-1 levels, the risk of thrombotic complications would be increased. Therefore, our study implies that lower TFPI-1 level may lead to the development and progression of the disorder with blood coagulation in NSCLC patients.

Importantly, cancer induced hemostatic activity is a trigger of increased thromboembolic events. On the other hand, DVT has been shown to promote tumor growth and cancer cell dissemination [22]. Some studies have suggested that hemostatic abnormalities observed in cancer patients may lead to the recruitment of inflammatory cells, the generation of tumor stroma, and angiogenesis [23]. For instance, tumors activating coagulation system are supposed to behave more aggressively with higher risk of invasion and metastasis. High levels of circulating biomarkers resembling activated coagulation and fibrinolytic system have been associated with 
decreased survival for several tumor types [24]. Those studies indicated that progression of disease condition of cancer patients is correlated with abnormal blood coagulation. In the present study, we found that there were significantly lower TFPI-1 levels in NSCLC patients either with DVT or with tumor metastasis than in those patients without DVT or tumor metastasis, respectively. In NSCLC patients with DVT or metastasis, lower TFPI-1 level may be due to consumption by tissue factor from tumor cells or damaged endothelial cells in ongoing coagulation activation. And lower TFPI1 levels in patients with metastasis may be explained by what TF-VIIa/TFPI-1 interactions which can promote tumor cell adhesion and migration [25]. Through the interactions, TFPI-1 may be partly involved in tumor metastasis and consumed, and lower TFPI-1 was consistent with these processes. Although we could not provide evidences to confirm the direct contribution of lower TFPI-1 level to DVT, the abovementioned significant correlation of decreased TFPI-1 with abnormal hemostatic markers had revealed that lower TFPI-1 level was also closely associated with the occurrence of DVT or tumor metastasis. Moreover, there was a high metastasis rate in patients with DVT and a higher DVT rate in patients with metastasis. Therefore, NSCLC patients with decreased TFPI-1 levels may be in risk for DVT and tumor metastasis, which indicates that TFPI-1 is useful to predict DVT and tumor metastasis at the time of diagnosis of NSCLC.

Various studies have demonstrated that D-D and other markers of coagulation and fibrinolytic activation are strong predictors of poor prognosis for lung cancer [20, 26, 27]. Therefore, the association between TFPI-1 and NSCLC may provide a possible explanation for NSCLC being closely related to DVT, even tumor metastasis. Based on this objective, we evaluated the significance of TFPI-1 for predicting DVT and tumor metastasis at the time of diagnosis of NSCLC. Multivariate regression analysis showed that TFPI-1 had a higher OR value in predicting DVT or metastasis than D-D, Fbg, AT, and FVIII in NSCLC patients, respectively, which indicated that TFPI-1 was a stronger risk factor of DVT and tumor metastasis and also further confirmed that decreased TFPI-1 levels might be correlated with DVT and tumor metastasis in NSCLC patients. Dahm et al. have reported that free TFPI levels below $9 \mathrm{ng} / \mathrm{mL}$ were associated with higher risk of DVT in patients with DVT but without known malignancies [28]. Though we only observed total TFPI-1 levels in our study, the findings may indicate the same clinical significance as above mentioned. To further assess the diagnostic power of TFPI-1, ROC curve analysis was used. From the ROC curve analysis, the area under ROC curve (AUC) of 0.884 and 0.856 demonstrated that TFPI- 1 is a valuable marker with higher predicting significance for DVT and metastasis at the diagnosis of NSCLC, respectively. And the cutoff values of $57.7 \mathrm{ng} / \mathrm{mL}$ and $54.3 \mathrm{ng} / \mathrm{mL}$ have a higher sensitivity and specificity in prediction of DVT and tumor metastasis, respectively. High D-D level can predict DVT, and it is also a good prognostic predictor for lung cancer patients [25]. Therefore, we further investigated whether the combination of TFPI-1 and D-D had a higher predictive value for DVT and metastasis in NSCLC patients. As expected, our results demonstrated a higher AUC of 0.928 and 0.878 , higher sensitivity of $87 \%$ and $85 \%$, and higher specificity of $79 \%$ and $70 \%$ for predicting DVT and metastasis, respectively, than those of single TFPI-1 measurements, indicating that combining TFPI-1 and D-D measurements may serve as more useful tool in prediction of DVT and metastasis of NSCLC patients.

There are some limitations concerning the present study. Firstly, TFPI-1 detection has no uniform standard and was widely used. We have measured TFPI-1 by ELISA in the study, and the result displayed a valuable clinical significance of TFPI-1 measurements. Furthermore, although we did not measure the direct markers of coagulation activation, we have found that TFPI-1 was significantly correlated to the indirect markers and closely associated with DVT and tumor metastasis, which revealed its clinical predicting values in lung cancer patients. Finally, only symptomatic or doubtful DVT or metastasis patients were included, and we cannot exclude that asymptomatic DVT or metastasis which was present in some patients and potentially decreased the predicting power of TFPI-1.

In conclusion, our study suggests that TFPI-1 is a valuable marker to predict the occurrence of DVT and tumor metastasis with a high sensitivity and specificity at the time of diagnosis of NSCLC patients. Further studies are required to assess whether TFPI- 1 could provide aid to the therapeutic decision-making for NSCLC patients.

\section{Competing Interests}

There was no conflict of interests.

\section{Acknowledgments}

The authors thank all persons who helped them gather information for patients and supported them for statistical analysis. This study was supported by the National Natural Science Foundation of China (Grant no. 81301406) and the Natural Science Foundation of Zhejiang Province (Grant no. LQ13H190005).

\section{References}

[1] S. Nomura, M. Niki, T. Tamaki, M. Shimizu, and T. Nishizawa, "Microparticles as biomarkers of blood coagulation in cancer," Biomarkers in Cancer, vol. 7, pp. 51-56, 2015.

[2] G. Radhakrishna and D. Berridge, "Cancer-related venous thromboembolic disease: current management and areas of uncertainty," Phlebology, vol. 27, no. 2, pp. 53-60, 2012.

[3] A. A. Khorana and G. C. Connolly, "Assessing risk of venous thromboembolism in the patient with cancer," Journal of Clinical Oncology, vol. 27, no. 29, pp. 4839-4847, 2009.

[4] H. Ogino, S. Hayashi, M. Kawasaki, M. Nakanishi, and N. Hara, "Association of thrombosis-inducing activity (TIA) with fatal hypercoagulable complications in patients with lung cancer," Chest, vol. 105, no. 6, pp. 1683-1686, 1994.

[5] H. Stricker, "Venous thromboembolism and cancer: pathophysiology and incidence," Vasa, vol. 43, no. 4, pp. 239-243, 2014. 
[6] G. Elyamany, A. M. Alzahrani, and E. Bukhary, "Cancerassociated thrombosis: an overview," Clinical Medicine Insights: Oncology, vol. 8, pp. 129-137, 2014.

[7] P. Girard, C. Raynaud, G. Meyer, F. Parent, and B. Besse, “Thrombosis and lung cancer," Revue de Pneumologie Clinique, vol. 64, pp. 85-91, 2008.

[8] E. Sierko, M. Z. Wojtukiewicz, L. Zimnoch, and W. Kisiel, "Expression of tissue factor pathway inhibitor (TFPI) in human breast and colon cancer tissue," Thrombosis and Haemostasis, vol. 103, no. 1, pp. 198-204, 2010.

[9] J. P. Wood, P. E. R. Ellery, S. A. Maroney, and A. E. Mast, "Biology of tissue factor pathway inhibitor," Blood, vol. 123, no. 19, pp. 2934-2943, 2014.

[10] C. Erem, M. Kocak, I. Nuhoglu, M. Yilmaz, and O. Ucuncu, "Increased plasminogen activator inhibitor-1, decreased tissue factor pathway inhibitor, and unchanged thrombin-activatable fibrinolysis inhibitor levels in patients with primary hyperparathyroidism," European Journal of Endocrinology, vol. 160, no. 5, pp. 863-868, 2009.

[11] S. A. Maroney and A. E. Mast, "New insights into the biology of tissue factor pathway inhibitor," Journal of Thrombosis and Haemostasis, vol. 13, supplement 1, pp. S200-S207, 2015.

[12] M. A. Kurer, "Protein and mRNA expression of tissue factor pathway inhibitor-1 (TFPI-1) in breast, pancreatic and colorectal cancer cells," Molecular Biology Reports, vol. 34, no. 4, pp. 221-224, 2007.

[13] V. Tagalakis, D. Levi, J. S. Agulnik, V. Cohen, G. Kasymjanova, and D. Small, "High risk of deep vein thrombosis in patients with non-small cell lung cancer: a cohort study of 493 patients," Journal of Thoracic Oncology, vol. 2, no. 8, pp. 729-734, 2007.

[14] S. Noble and J. Pasi, "Epidemiology and pathophysiology of cancer-associated thrombosis," British Journal of Cancer, vol. 102, supplement 1, pp. S2-S9, 2010.

[15] A. Falanga, M. Marchetti, and A. Vignoli, "Coagulation and cancer: biological and clinical aspects," Journal of Thrombosis and Haemostasis, vol. 11, no. 2, pp. 223-233, 2013.

[16] A. Falanga, M. Marchetti, and L. Russo, "The mechanisms of cancer-associated thrombosis," Thrombosis Research, vol. 135, supplement 1, pp. S8-S11, 2015.

[17] T. O. Königsbrügge, I. Pabinger, and C. Ay, "Risk factors for venous thromboembolism in cancer: novel findings from the Vienna Cancer and Thrombosis Study (CATS)," Thrombosis Research, vol. 133, supplement 2, pp. S39-S43, 2014.

[18] Y. Zhu, Q.-W. Cong, Y. Liu et al., "Antithrombin, an important inhibitor in blood clots," Current Topics in Medicinal Chemistry, vol. 16, no. 6, pp. 666-674, 2016.

[19] A. E. Mast, "Tissue factor pathway inhibitor: multiple anticoagulant activities for a single protein," Arteriosclerosis, Thrombosis, and Vascular Biology, vol. 36, no. 1, pp. 9-14, 2016.

[20] F. Tas, L. Kilic, M. Serilmez, S. Keskin, F. Sen, and D. Duranyildiz, "Clinical and prognostic significance of coagulation assays in lung cancer," Respiratory Medicine, vol. 107, no. 3, pp. 451-457, 2013.

[21] N. Iversen, A.-K. Lindahl, and U. Abildgaard, "Elevated TFPI in malignant disease: relation to cancer type and hypercoagulation," British Journal of Haematology, vol. 102, no. 4, pp. 889895, 1998.

[22] A. Amirkhosravi, T. Meyer, M. Amaya et al., "The role of tissue factor pathway inhibitor in tumor growth and metastasis," Seminars in Thrombosis and Hemostasis, vol. 33, no. 7, pp. 643652, 2007.
[23] F. R. Rickles, M. N. Levine, and H. F. Dvorak, "Abnormalities of hemostasis in malignancy," in Hemostasis and Thrombosis. Basic Principles and Clinical Practice, R. W. Colman, J. Hirsh, V. J. Marder, A. W. Clowes, and J. N. George, Eds., p. 1131, Lippincott Williams and Wilkins, Philadelphia, Pa, USA, 4th edition, 2001.

[24] S. Oya, Y. Akiyama, T. Okuyama, and H. Ishikawa, "High preoperative plasma $\mathrm{D}$-dimer level is associated with advanced tumor stage and short survival after curative resection in patients with colorectal cancer," Japanese Journal of Clinical Oncology, vol. 31, no. 8, pp. 388-394, 2001.

[25] E. G. Fischer, M. Riewald, H.-Y. Huang et al., "Tumor cell adhesion and migration supported by interaction of a receptorprotease complex with its inhibitor," The Journal of Clinical Investigation, vol. 104, no. 9, pp. 1213-1221, 1999.

[26] M. Franchini, M. Montagnana, G. Targher, F. Manzato, and G. Lippi, "Pathogenesis, clinical and laboratory aspects of thrombosis in cancer," Journal of Thrombosis and Thrombolysis, vol. 24, no. 1, pp. 29-38, 2007.

[27] C. Ay, D. Dunkler, R. Pirker et al., "High D-dimer levels are associated with poor prognosis in cancer patients," Haematologica, vol. 97, no. 8, pp. 1158-1164, 2012.

[28] A. Dahm, A. van Hylckama Vlieg, B. Bendz, F. Rosendaal, R. M. Bertina, and P. M. Sandset, "Low levels of tissue factor pathway inhibitor (TFPI) increase the risk of venous thrombosis," Blood, vol. 101, no. 11, pp. 4387-4392, 2003. 


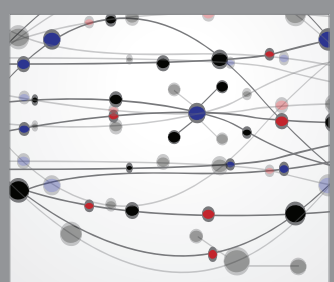

The Scientific World Journal
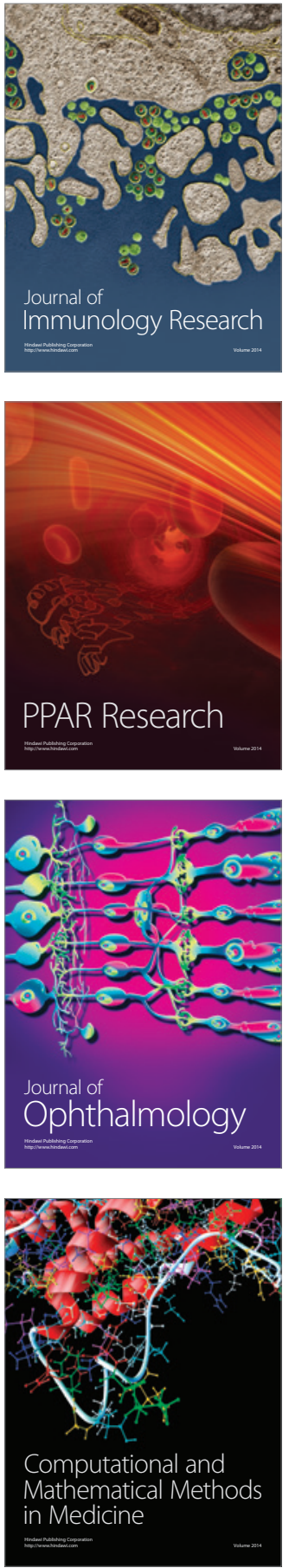

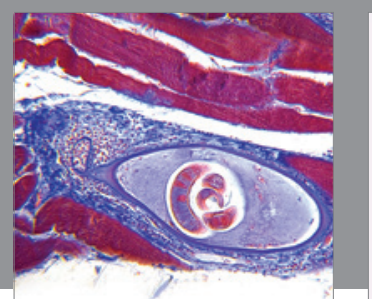

Gastroenterology Research and Practice
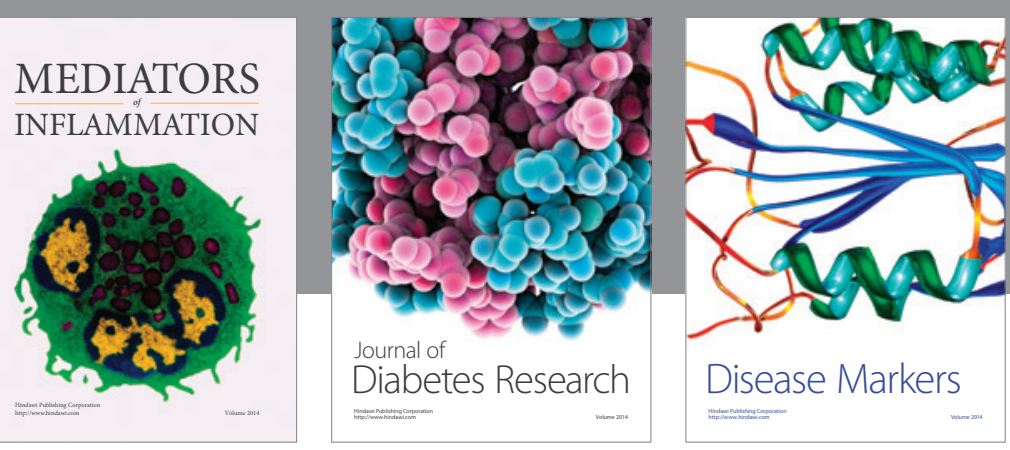

Disease Markers

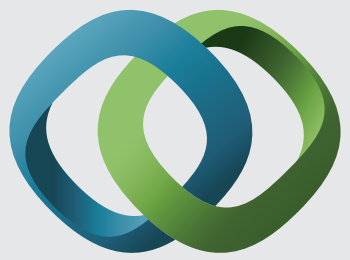

\section{Hindawi}

Submit your manuscripts at

https://www.hindawi.com
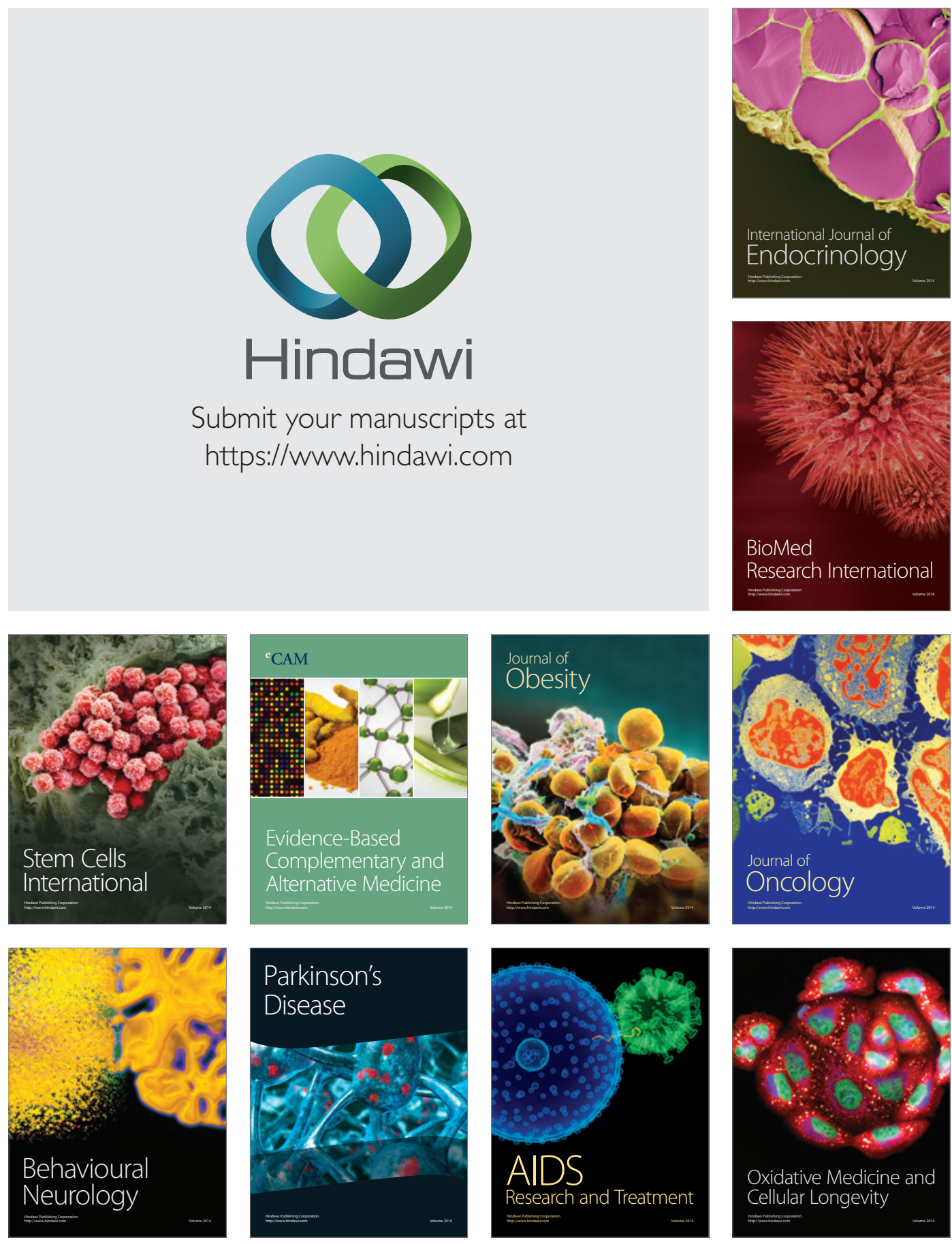